\title{
Oblique shock reflection from an axis of symmetry
}

\author{
By H. G. HOR N UNG \\ Graduate Aeronautical Laboratories, California Institute of Technology, Pasadena, CA 91125, USA
}

(Received 11 November 1998 and in revised form 31 March 1999)

An exploratory computational study of the reflection of an inward-facing conical shock wave from its axis of symmetry is presented. This is related to more complex practical situations in both steady and unsteady flows. The absence of a length scale in the problem studied makes features grow linearly with time. The ensuing flow is related to the Guderley singularity in a cylindrical imploding shock. The problem is explored by making a large number of computations of the Euler equations. Distinct reflection configurations are identified, and the regions of parameter space in which they occur are delineated.

\section{Introduction}

This study started with a computational investigation of a process that occurs in a shock tunnel. The idea of a shock tunnel is to produce a high-speed gas flow for a short time. The high speed is achieved by expanding the gas from a high-enthalpy reservoir state through a nozzle. To bring the gas to the required reservoir state, it is heated and compressed by propagating a shock wave through it in a shock tube, and reflecting the shock wave from the endwall of the shock tube, so that heating and compression of the gas is performed by both the incident and the reflected shock. The endwall has a hole in it that serves as the nozzle throat and is initially closed by a thin diaphragm that bursts when the incident shock arrives. The problem is that the shock reflection at the throat produces a convex shock that converges onto the axis of symmetry of the throat.

Figure 1 illustrates the process with a computed time sequence. The incident shock is reflected from the area reduction, forming the convex converging shock which is reflected from the axis. As the angle at which the shock intersects the axis increases, the reflection produces a strong jet to the left. This creates a vortex ring that is strong enough to maintain a bulge in the reflected shock and a disk-shaped shock within it. (It is this strong vortex ring that provides me with a tenuous connection to Philip Saffman's work, though I am not quite sure whether he believed its existence when I presented it in a seminar at Caltech). Such a strong vortex ring is clearly not desirable in a reservoir, where one wishes the flow to be as uniform as possible. Changing the radius of curvature of the throat entrance makes no difference to this phenomenon, which is plausible, since the convergence of the shock onto the axis is virtually independent of the radius.

The observation of the strong vortex ring is not new. It has been discussed by Zabusky et al. (1997), who called it the "vortex ring projectile". They observed it in a different situation that occurs when a shock wave passes over a heavy spherical bubble surrounded by a lighter gas. The diffracted shock becomes convex 

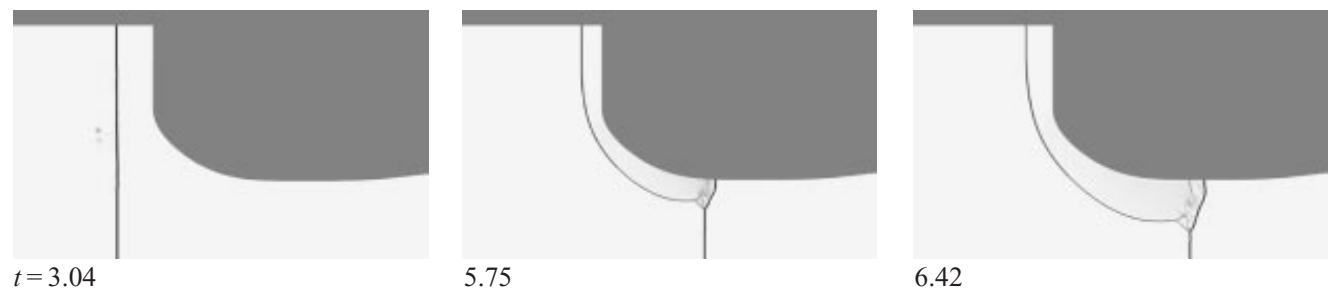

5.75

6.42
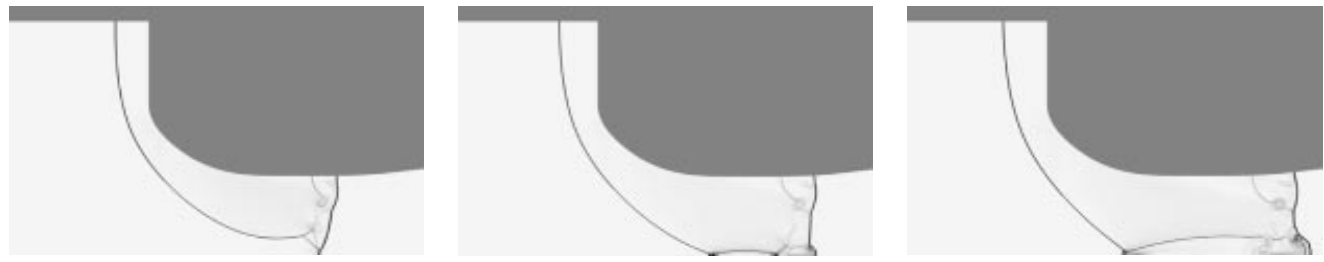

7.07

7.60

8.17

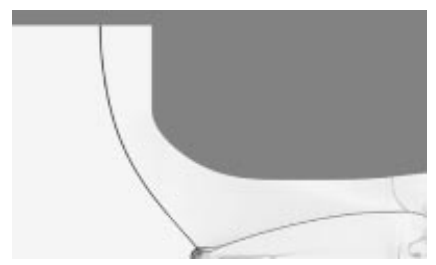

9.12

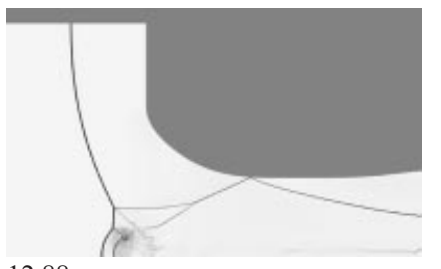

12.88

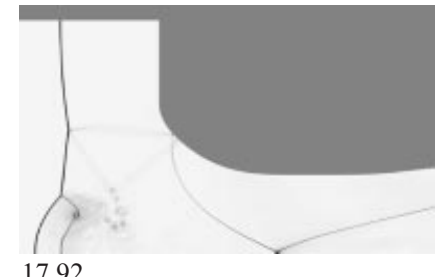

17.92
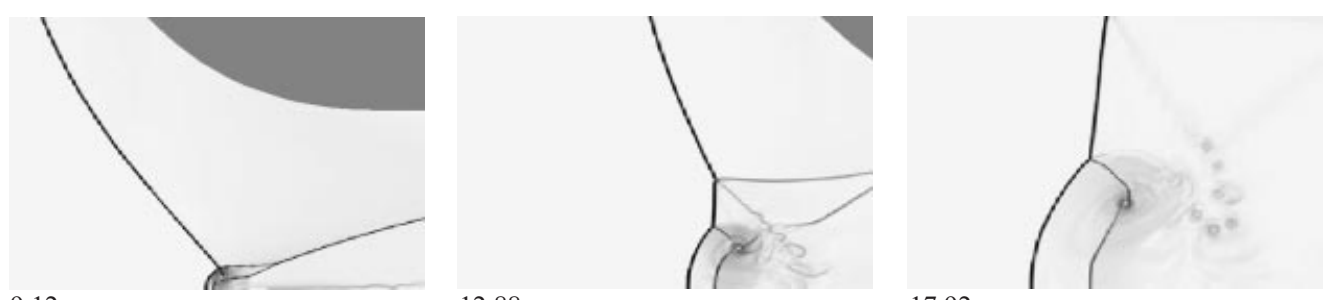

17.92

FIGURE 1. Computed time sequence of the shock reflection process in the shock tunnel. The greyshading in the images is a monotonic function of the magnitude of the density gradient scaled by the local density. $M_{\mathrm{s}}=9, \gamma=1.2$. The symmetry axis lies along the bottom edge of the pictures. Note the strong vortex ring in the ninth frame, with a shock in its centre. The bottom row shows enlarged views of the third row. The computation solves the Euler equations in a body-fitted grid of 14400 coarse cells with two tiers of adaptive mesh refinement by a factor of 3 each, so that the effective number of cells is 1166400 .

and converges onto the symmetry axis at the rear of the bubble, reflecting from the axis and forming a vortex ring just as in the shock tunnel. This process is illustrated in a computed time sequence in figure 2. Similar convex shock implosions were also studied experimentally and theoretically by Barkhudarov et al. (1989). The waves were relatively weak, however, which may be the reason why the vortex ring was not observed.

In these flows, there exists a brief time interval just as the convex shock reaches the axis, during which conditions are locally like those in the implosion of a cylindrical shock onto its axis, which is a one-dimensional unsteady flow that has a self-similar solution found by Guderley, see Whitham (1974), with a power-law singularity at the axis. This singularity cannot be resolved by the computation, so that the possibility 

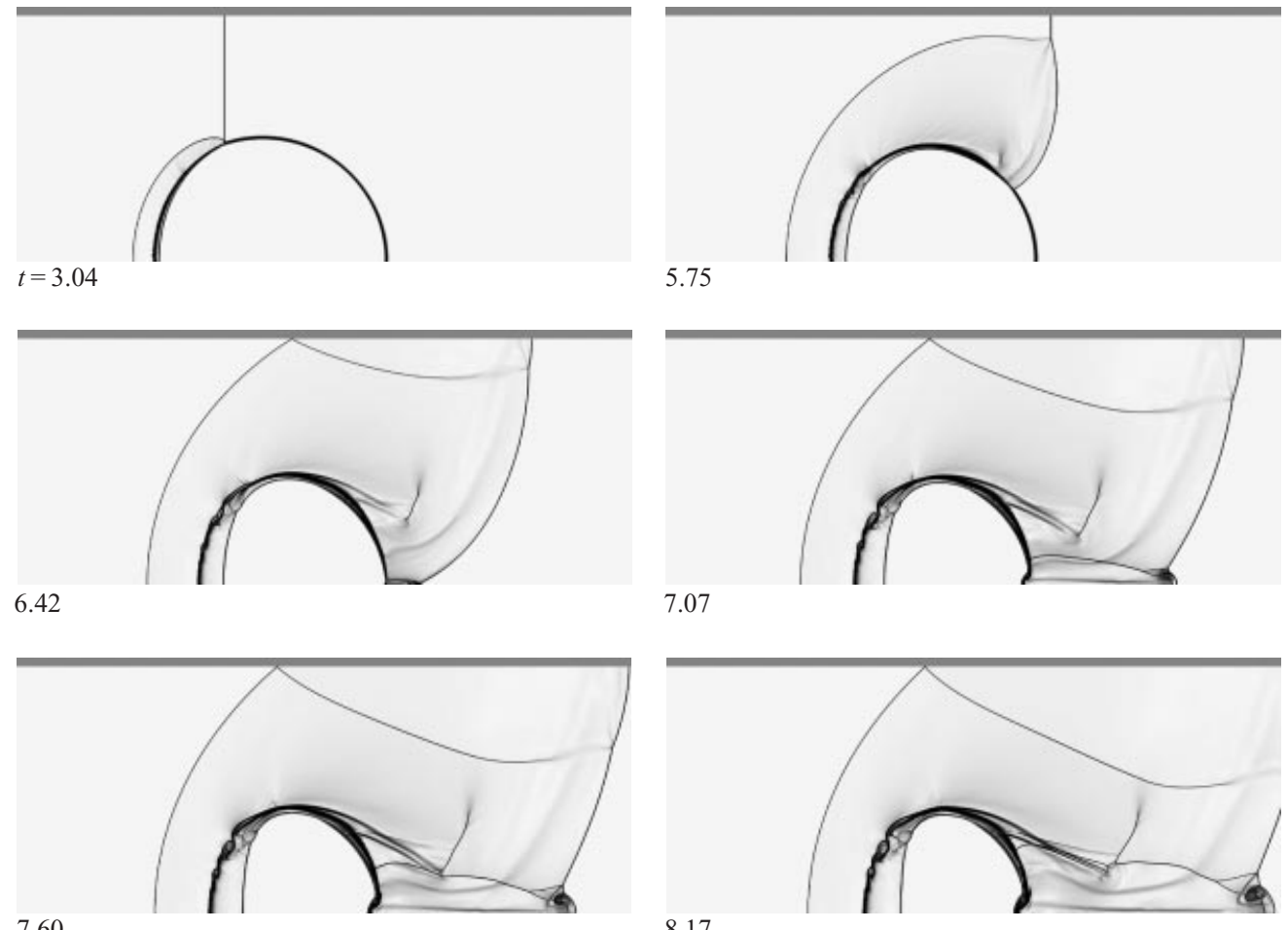

FIGURE 2. Time sequence of shock diffraction over a heavy spherical bubble. Shock Mach number $=10$, specific heat ratio $=1.4$, density ratio $=100$. Note the strong vortex ring at the bottom right of the last frame. The symmetry axis lies along the bottom edge of the pictures.

exists that the strong vortex ring is an artifact of the computation method. On the other hand, the cylindrical implosion is known to be unstable, see Whitham (1974). Small perturbations of a circular shock grow as the shock converges onto the axis, so that it becomes polygonal, with the result that the amplification due to focusing is much more benign than the self-similar solution predicts. This has also been observed experimentally, e.g. by Takayama, Kleine \& Grönig (1987). In computations that force the flow to be axisymmetric, such as those of figures 1 and 2, this instability, which may conceivably prevent the formation of the vortex ring in physical reality, is, of course, impossible.

In the shock tunnel the singularity can be avoided by placing a rod along the axis, so that the shock is reflected off a body of finite diameter rather than from the axis. Figure 3 shows a frame corresponding to the last frame of figure 1 when the setup includes such a rod. It is clear that the rod prevents the vortex ring from being formed. The question is whether this is because the singularity is avoided or because the detail of the early reflection is now better resolved. In fact, in this example, the vortex ring disappears completely only for rod diameters of 12 coarse grid cells or more, which suggests that it is not caused by lack of resolution.

There exists a steady-flow analogue of the unsteady one-dimensional implosion problem. This occurs, e.g. in an axisymmetric, overexpanded, supersonic jet, in which an inward-facing conical shock is formed at the nozzle exit. In this case, the shock usually reflects off the axis as a Mach reflection. A related problem was studied 


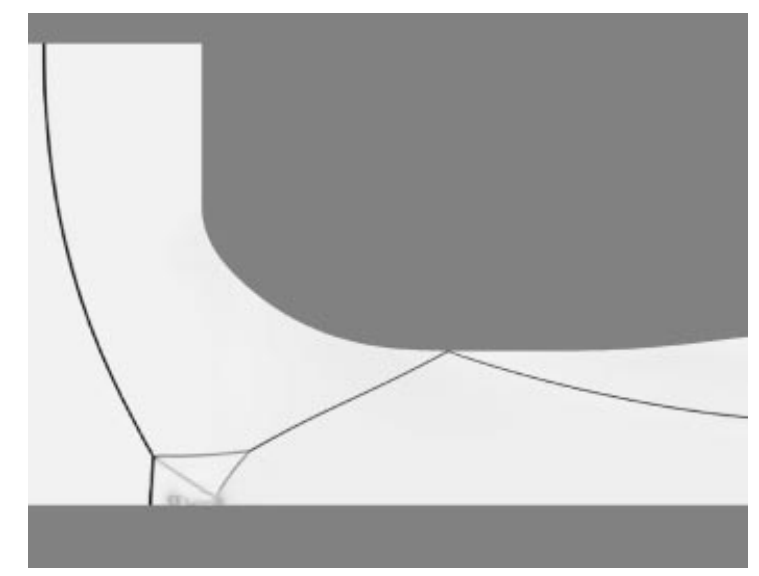

FIGURE 3. Shock reflection at the endwall of the shock tube in a shock tunnel with a rod along the axis. The rod diameter in this flow is the smallest for which the vortex ring disappears. The symmetry axis lies along the bottom edge of the picture. Conditions otherwise are as in figure 1 .

in a hypersonic gun tunnel by Mölder et al. (1997), who observed that the shock steepens with decreasing radius, until the shock angle reaches the von Neumann angle (the smallest angle at which Mach reflection of strong shocks is possible). At this point, transition to Mach reflection occurs. The shock was generated by a ring that was shaped, according to an extension of the Taylor-Maccoll theory to shock angles larger than $90^{\circ}$, in order to produce an inward-facing conical shock. When the shock was weak at the lip of the ring, the steepening could not be detected in the experiment. However, in highly refined computations, a tiny Mach reflection was found, preceded by steepening. For two stronger shocks, the size of the Mach reflection was appropriately larger and was easily detected. The authors had initially expected their special ring shapes to produce straight conical shocks. In fact, the regular reflection implied by such a conical shock is not possible, as has been shown theoretically by Rylov (1990). The steepening seems to be analogous to the strengthening of the imploding shock, with a singular, power-law shock shape near the axis, the singularity being prevented by the occurrence of a Mach reflection as soon as the von Neumann angle is reached.

All of these interesting flows seem to be related to the Guderley singularity in some way. Also, the singularity is prevented by the occurrence of Mach reflection in the steady-flow problem, and may be prevented by axial unsymmetry (threedimensionality) or by Mach reflection in the convex shock implosion. Unfortunately none of them is a particularly simple problem. A clean problem can, however, be abstracted, that has a bearing on both the shock tunnel problem and the Mölder flows. Imagine a diaphragm in the shape of a cone. Let the pressure on the outside of the cone be high and the pressure inside the cone be low. Let the diaphragm burst instantaneously, so that a shock wave propagates into the low-pressure gas inside the cone, and an expansion propagates into the high-pressure gas on the outside. The shock strength is initially uniform. As time proceeds and the shock is reflected from the axis, the features of this flow grow linearly in time, since the problem does not possess a length scale (inviscid perfect-gas flow). This pseudo-steady problem is the subject of the remainder of this study. It has some similarities with the flow that occurs in a shaped charge. 


\section{Specification of the problem}

The flow is assumed to be inviscid and axisymmetric, and the medium is taken to be a perfect gas. We therefore compute the flow using the unsteady Euler equations. Actually, since the flow is expected to be self-similar, the Euler equations could be transformed from physical space, $(\boldsymbol{x}, t)$, to pseudo-steady space, $(\boldsymbol{x} / t, t)$, with the result that the explicit time dependence cancels out. The solution could then be obtained by solving the transformed equations, see e.g. Samtaney \& Pullin (1996). This is more computationally intensive, however, and we choose to solve the unsteady Euler equations.

\subsection{Initial conditions}

Imagine a three-dimensional physical space to be divided into two regions by a conical surface. The apex of the cone is at $x=0$, and the positive $x$-direction is the symmetry axis of the cone and forms an acute angle $\alpha$ with the cone's generators. Let the space on the positive $x$-axis side of the surface be called the inside of the cone. All of the space is filled with the same perfect gas. At times $t<0$, constrain the pressure and temperature on the outside to $p_{4}, T_{4}$, and on the inside to $p_{1}, T_{1}$. At $t=0$, remove the constraint instantaneously and let the flow evolve.

If we do the computation in a Cartesian grid, the conical interface cuts the cells at an angle, so that the boundary between the two gas states is represented by a staircase shape instead of the sharp straight line we want. The computation can handle this at relatively low pressure ratios, but breaks down in the more interesting high pressure ratio cases. To avoid this problem, we start the flow off with an interface of finite thickness, across which the temperature and pressure have hyperbolic tangent profiles. The steepest-slope thickness of the interface is approximately 2 coarse-grid cells.

\subsection{The computational setup}

For the computations, the software system Amrita, constructed by James Quirk, see Quirk (1998), was used. Amrita is a system that automates and packages computational tasks in such a way that the packages can be combined (dynamically linked) according to instructions written in a high-level scripting language. The present application uses features of Amrita that include the automatic construction of different Euler solvers, automatic documentation of the codes, automatic adaptive mesh refinement according to simply chosen criteria, and scripting-language-driven computation, archiving and post-processing of the results. The automation of the assembly and sequencing of the tasks dramatically reduces the possibility of hidden errors. More importantly, it makes computational investigations transparent and testable by others. The ability to change one package at a time, without changing the rest of the scheme, permits easy detection of sources of error. The scope of the software system far exceeds its use here. The Euler solver generated for the present computations was an operator-split scheme with HLLE flux and kappa-MUSCL reconstruction.

\section{Results}

\subsection{Choice of parameter space}

In a perfect gas, the pseudo-steady behaviour of the shock wave is determined by the initial shock Mach number, $M_{\mathrm{s}}$, the angle $\alpha$ and the ratio of specific heats, $\gamma$. The flow is also affected, however, by the ratio of sound speeds across the interface, since the shock that is reflected from the axis impinges onto the interface, and the 


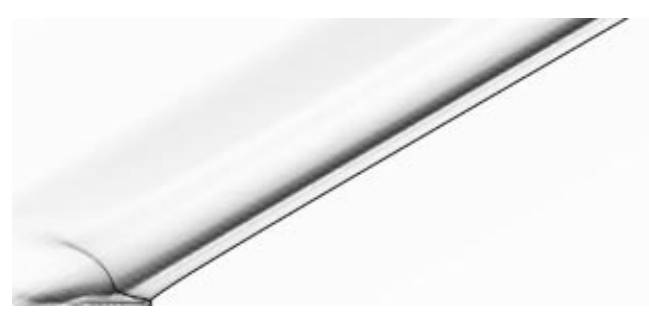

FIGURE 4. To illustrate the features of the process, we show an early phase of the oblique shock implosion shortly after the conical diaphragm has burst. The dispersed expansion wave that propagates to the upper left is visible as a broad grey band. The shock wave that propagates to the bottom right, toward the axis of symmetry is seen as a sharp black line followed by the black contact surface just above it. The faint white line marks the original location of the diaphragm.

$\begin{array}{ccccccc}p_{4} / p_{1} & 2 & 5 & 10 & 20 & 50 & 100 \\ \gamma & & & & & & \\ 1.2 & 1.20 & 1.67 & 2.23 & 3.05 & 4.73 & 6.65 \\ 1.4 & 1.19 & 1.62 & 2.15 & 2.93 & 4.52 & 6.34\end{array}$

TABLE 1. Values of $M_{\mathrm{s}}$ at computed conditions with $T_{4} / T_{1}=p_{4} / p_{1}$.

details of this interaction are sensitive to this ratio. In order to cover the parameter space with a reasonable density we choose conditions according to table 1 with $T_{4} / T_{1}=p_{4} / p_{1}$, for the shock angles $\alpha=20^{\circ}, 30^{\circ}$ and $40^{\circ}$. Additional computations were done with different temperature ratios, but the range of table 1 suffices for the present discussion.

\subsection{The reflection configurations}

The main features of the early evolution of the flow may be seen in the pseudoschlieren image of figure 4. Six later stage images are shown in figure 5 of the results of computations with $\alpha=20^{\circ}$ for shock strengths ranging from $M_{\mathrm{s}}=1.19$ to 6.34 . In the case of the weakest shock, the incident shock may be seen to steepen as expected; however, the reflection configuration seems to be regular. At $M_{\mathrm{s}}=1.62$ (top right), the characteristic wake after a Mach reflection can be distinguished. As the shock strength is increased, the shock steepening becomes more pronounced, the Mach reflection becomes more easily recognizable, and the interface follows the shock more closely and becomes sharper. The interaction between the reflected shock and the interface is determined by the speed-of-sound ratio across it, i.e. by the temperature ratio. This is why the character of the transmitted wave changes in these examples, for which the temperature ratio has been coupled with the pressure ratio. In all of these frames, the expansion wave that propagates into the driver gas to the top left has already left the field of view.

In order to examine the reflection configurations in these cases more closely, figure 6 shows enlarged views of the same cases. Now, the Mach stem in all but the weakest-shock case can be distinguished clearly. The resolution of the computation in that case is not good enough to detect it. The character of the reflection seems to change at the higher Mach numbers. We will return to this after discussing the larger angles.

Figure 7 shows similar views of the case $\alpha=30^{\circ}$. In all of these, the steepening of the incident shock is evident. Also, the first three clearly exhibit simple Mach 

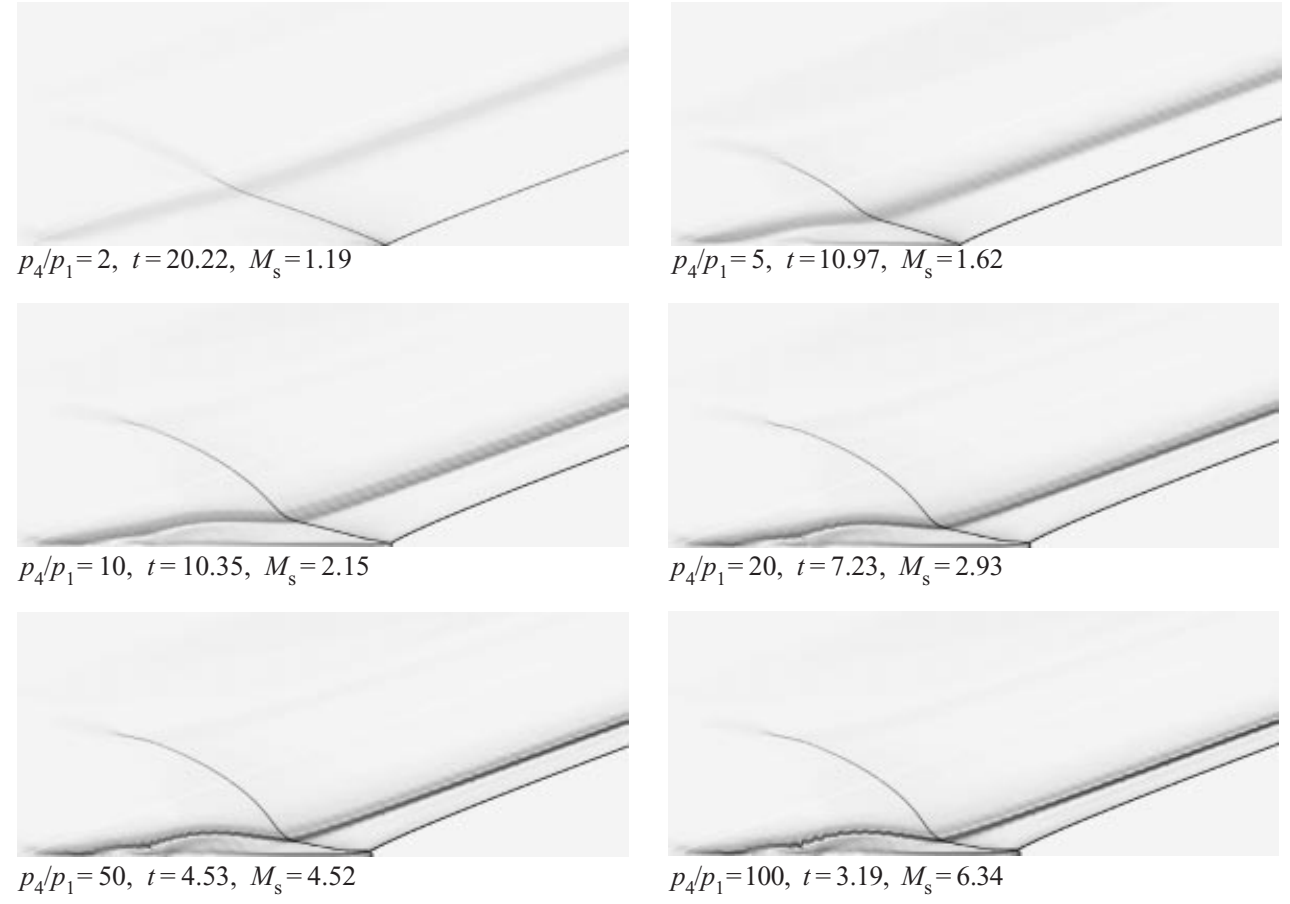

FIGURE 5. Dependence of the reflection configuration on shock strength for the case of $\alpha=20^{\circ}$, $T_{4} / T_{1}=p_{4} / p_{1}, \gamma=1.4$. The thin white line marks the original location of the diaphragm. The waviness of the interface in the stronger-shock cases is a memory of the initial representation of the interface in the coarsest grid.

reflection with a straight Mach stem. However, in the case $M_{\mathrm{s}}=2.93$, as in the stronger ones, the Mach stem shows a strong forward bulge, and a reflected shock that is almost parallel to the axis. In addition, another shock follows the reflected shock and is almost parallel to it.

In figure $8, \alpha=40^{\circ}$, the Mach stems are larger, so that the structure of the reflection configuration can be more easily resolved. It now becomes clear that the bulge of the Mach stem appearing for the higher shock strengths is associated with a vortex ring that seems to be the same phenomenon as observed in the shock tunnel problem and in the diffraction of a shock over a heavy bubble. At $M_{\mathrm{s}}=2.93$ the vortex ring causes a second triple point to appear on the Mach stem. With increasing shock (and vortex ring) strength, the second triple point moves up along the Mach stem. In calculations for even higher shock strengths, the second triple point eventually merges with the main triple point, producing the configuration seen in the case of the strong shocks with $\alpha=30^{\circ}$. Returning to figure 5, the barely resolved strong-shock reflections may now be recognized as this same configuration. Again, the strongest shocks produce reflected shocks that are nearly parallel to the symmetry axis, which are followed by another shock.

The interaction of the reflected shock with the interface now produces a transmitted wave with a propagation velocity component parallel to the interface that is faster than the intersection of the reflected wave and the interface. This causes a signal to be retransmitted across the interface into the test gas, where this precursor wave is supersonic. 


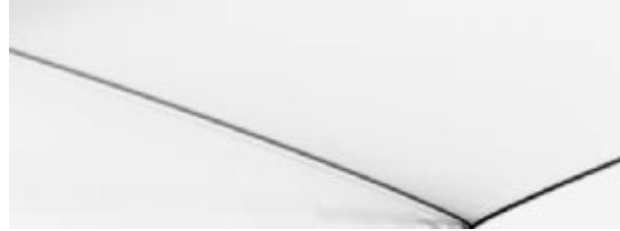

$p_{4} / p_{1}=2, t=20.22, M_{\mathrm{s}}=1.19$

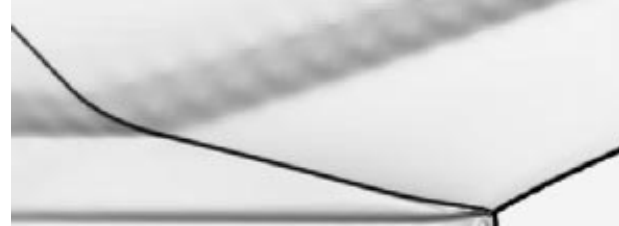

$p_{4} / p_{1}=10, t=10.35, M_{\mathrm{s}}=2.15$

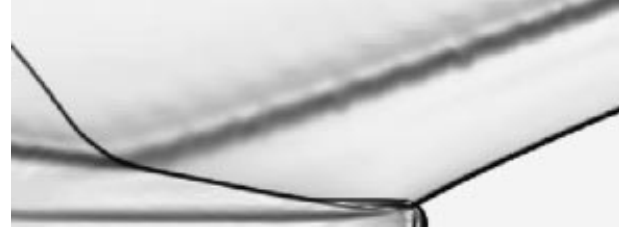

$p_{4} / p_{1}=50, t=4.53, M_{\mathrm{s}}=4.52$
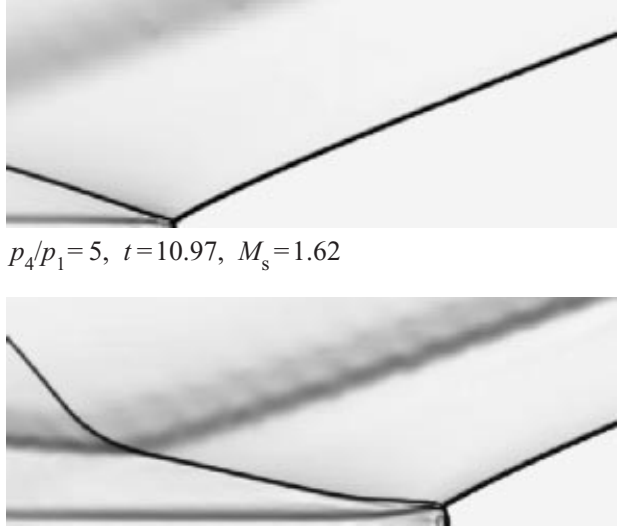

$p_{4} / p_{1}=20, t=7.23, M_{\mathrm{s}}=2.93$

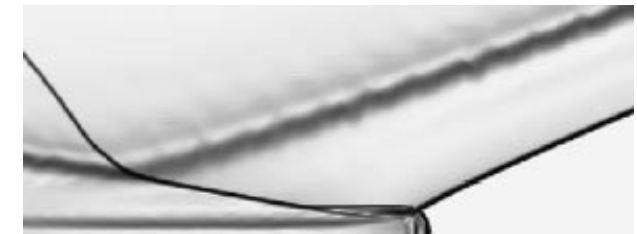

$p_{4} / p_{1}=100, t=3.19, M_{\mathrm{s}}=6.34$

FIGURE 6. Dependence of the reflection configuration on shock strength for the case of $\alpha=20^{\circ}$, $T_{4} / T_{1}=p_{4} / p_{1}, \gamma=1.4$. Enlarged views of the shock reflection structure.

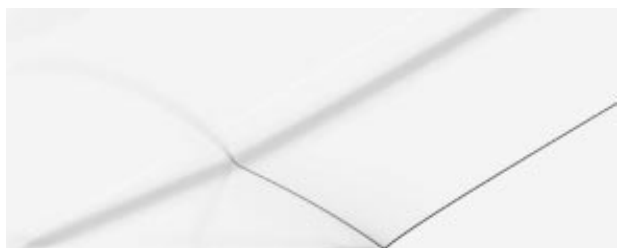

$p_{4} / p_{1}=2, t=29.99, M_{\mathrm{s}}=1.19$
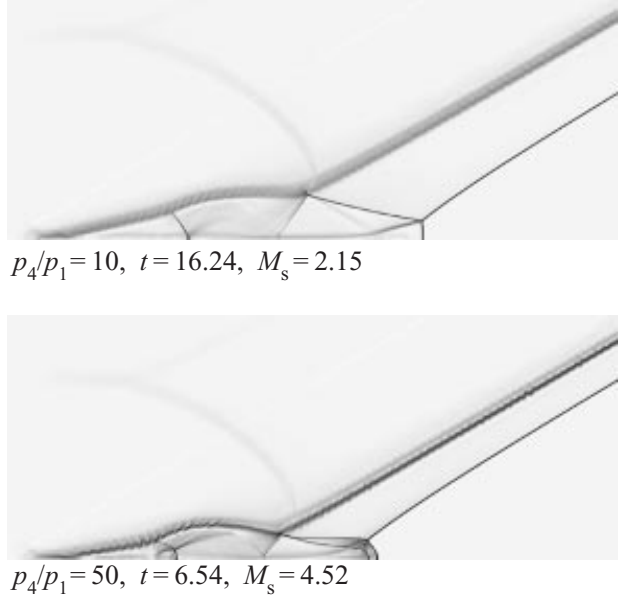
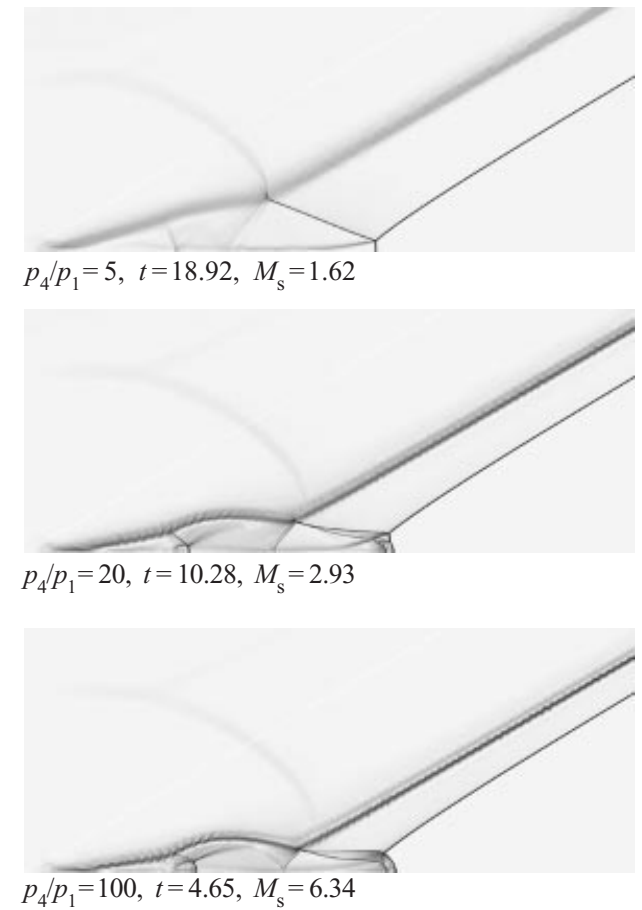

FIGURE 7. Dependence of the reflection configuration on shock strength for the case of $\alpha=30^{\circ}$, $T_{4} / T_{1}=p_{4} / p_{1}, \gamma=1.4$ 

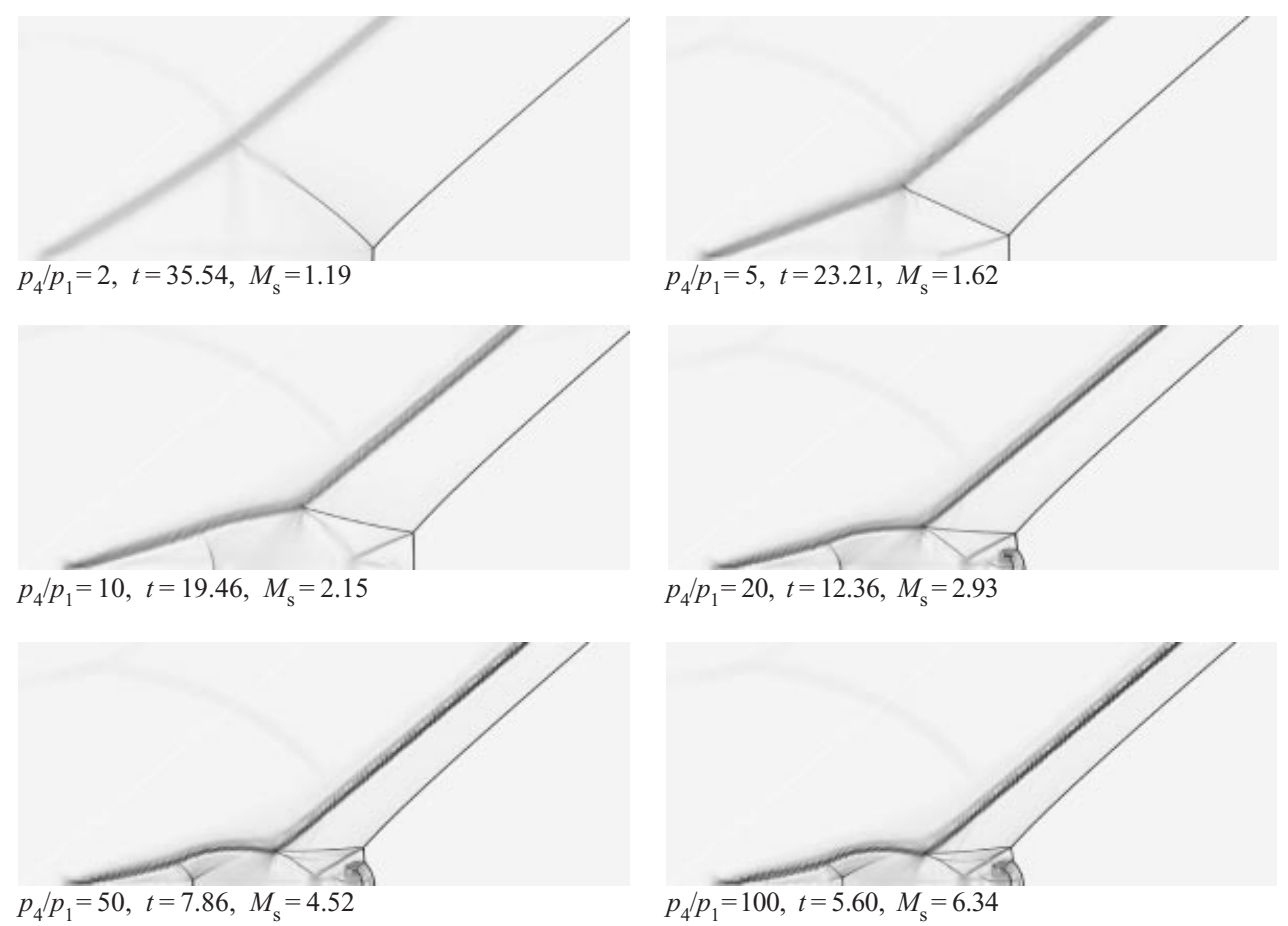

FIGURE 8. Dependence of the reflection configuration on shock strength for the case of $\alpha=40^{\circ}$, $T_{4} / T_{1}=p_{4} / p_{1}, \gamma=1.4$.

\subsection{Test of self-similarity}

The absence of a characteristic length in this problem should manifest itself in configurations that grow linearly with time. In the computation, we are, of course never free of a length scale. In the present case, in addition to the grid scale, we have introduced a finite initial interface thickness. In order to test the quality of the self-similarity of the solutions obtained here, figure 9 shows three enlarged views of the reflection configuration at $M_{\mathrm{s}}=2.93$ for the three angles. In these, an early phase of the flow is shown as a pseudo-schlieren image. Then a later phase is superimposed onto this picture in the form of contours of density gradient at a scale corresponding to that appropriate for linear growth, using white lines. It appears that the detailed features of the configurations are just about congruent, though the thickness of the interface clearly becomes relatively smaller at the later times. This is a scale effect related to the initial interface thickness.

However, on close inspection of the $20^{\circ}$ case, the Mach stem in the late phase is seen to be ahead of that of the early phase by about two shock thicknesses. Inadequate resolution of the features in the early phase is clearly responsible for this difference, which is thus a scale effect related to the grid scale.

\subsection{Approximate boundaries of the reflection configurations}

Enough of the parameter space has been covered by the computations shown here and additional ones not shown to draw approximate boundaries between the three main reflection configurations observed: the simple Mach reflection (S), the bulging Mach stem with two triple points (DV), and the vortical Mach reflection (V) in which the vortex ring dominates the Mach stem. This has been done in figure 10 in a plot 

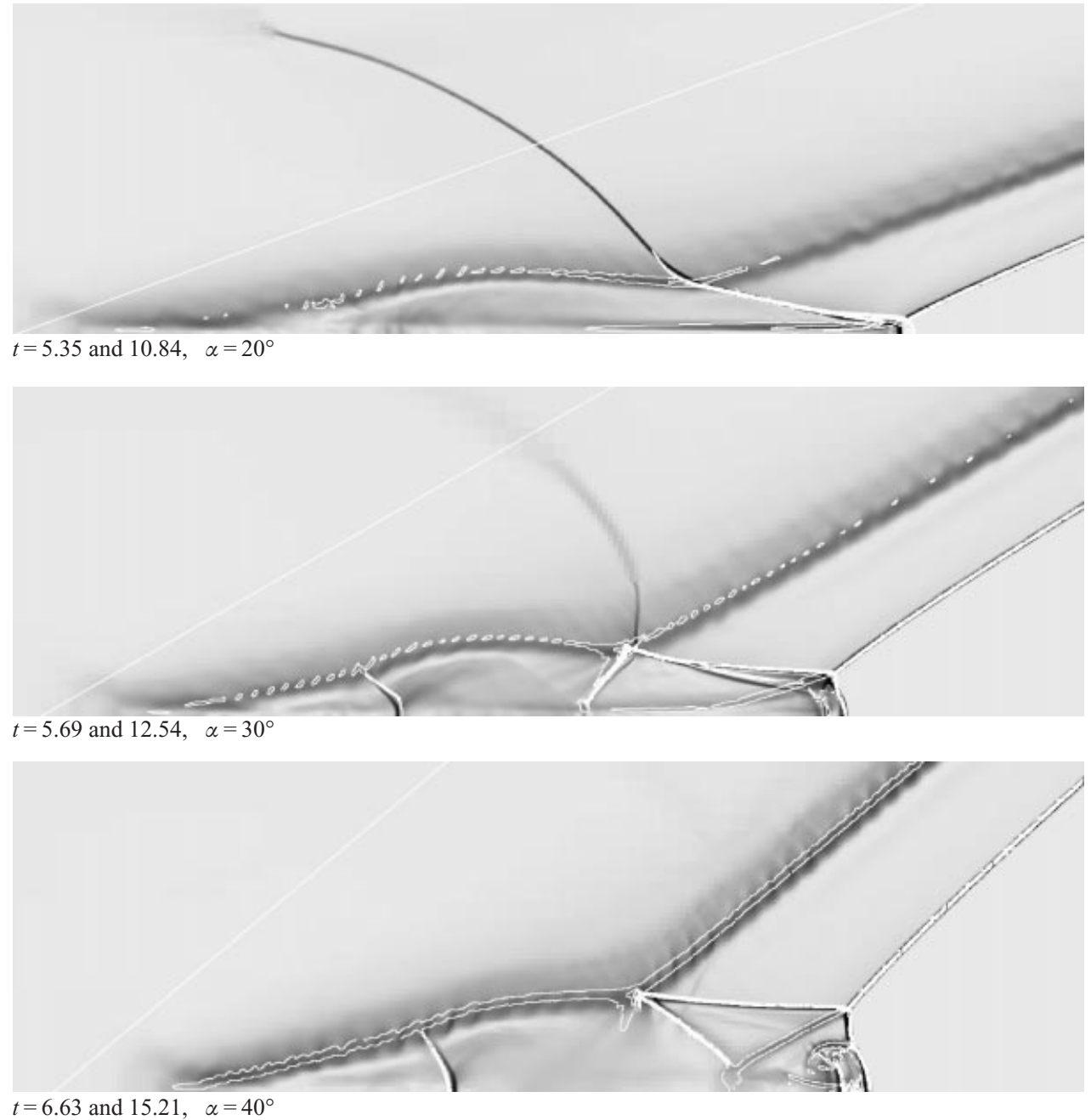

FIGURE 9. Test of self-similarity, $M_{s}=2.93, \gamma=1.4$. The white lines are contours of density gradient for a later time, scaled according to the time.

of $\alpha$ against $M_{\infty}=M_{\mathrm{s}} / \sin \alpha$ for $\gamma=1.2$ and 1.4. The wide region of the occurrence of DV in the case of larger angle when $\gamma=1.4$ all but disappears when $\gamma=1.2$. The sensitivity of reflection configurations to $\gamma$ is a phenomenon that occurs also in plane shock reflection.

\subsection{Comparison with shock-tunnel and bubble flows}

Returning to the sequence shown in figure 1, we can now recognize the reflection configurations that occur there as fitting in with the behaviour of the abstracted problem, in as much as the $\mathrm{V}$ configuration is observed immediately after the convex shock hits the axis until the intersection of the shock with the axis increases, so that, between frames 7 and 8, the transition to DV occurs. The same transition is observed in figure 2, between frames 4 and 5 . 

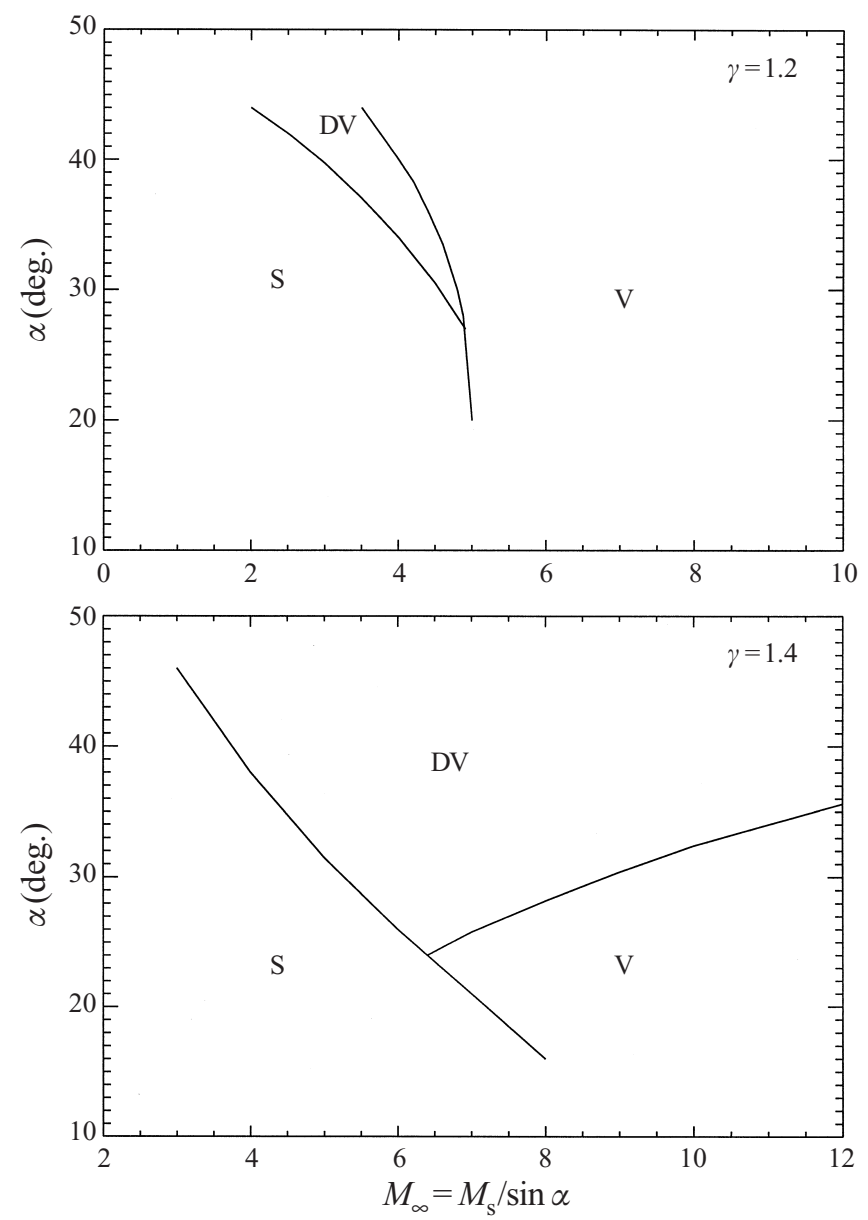

FIGURE 10. Approximate boundaries between the three reflection configurations for $\gamma=1.2$ and 1.4.

\section{A possible physical realization}

A conical diaphragm separating gases at different pressure is not a very easy thing to realize experimentally. Instead, one could generate the self-similar flow by shaping a solid explosive in the form of a hollow cone and detonating it from the cone surface.

In a limited parameter range, an easier method would be to shape the endwall of a shock tube like a hollow cone, at an angle such that the shock is reflected in a regular reflection from the cone surface. This reflected shock is then initially conical and has uniform strength between the cone apex and the point reached by the corner signal. This converging conical shock will then reflect off the axis just as in the case studied here. Unfortunately, the parameter range that can be covered in this way is restricted to large values of $\alpha$.

\section{Conclusions}

An exploratory computational study has been made of the Mach reflection configurations that occur when a conical shock reflects from its axis of symmetry. Distinct reflection configurations are identified and the regions of parameter space in which they occur are delineated. The inviscid problem has no characteristic length so that 
features grow linearly with time. The manner in which a practical problem led to the abstraction of the self-similar one is explained, and its features are related to those of the Guderley singularity and to a steady-flow analogue. In a sequel to this exploratory work shock steepening and the genesis of the reflection configurations will be discussed.

I wish to thank Dale Pullin and Gerry Whitham for valuable discussions and James Quirk for very important help with the computations.

\section{REFERENCES}

Barkhudarov, E. M., Mdivinshvill, M. O., Sokolov, I. V., Taktakishvili, M. I. \& Terekhin, V. E. 1989 Mach reflection of a ring shock wave from the axis of symmetry. J. Fluid Mech. 226, 497-509.

Mölder, S., Gulamhussein, A., Timofeev, E. \& Voinovich, P. 1997 Focusing of conical shocks at the center-line of symmetry. In Shock Waves (ed. A. F. P. Houwing \& A. Paull), pp. 875-880. Panther Publishing and Printing, Canberra.

Quirk, J. J. 1998 Amrita - a computational facility (for cfd modelling). VKI 29th CFD Lecture Series, ISSN 0377-8312.

RYLOV, A. I. 1990 On the impossibility of regular reflection of a steady-state shock wave from the axis of symmetry. Prikl. Mat. Mech. 54, 200-203.

Samtaney, R. \& Pullin, D. I. 1996 Initial-value and self-similar solutions of the compressible Euler equations. Phys. Fluids 8, 3072-3084.

Takayama, K., Kleine, H. \& Grönig, H. 1987 An experimental investigation of the stability of converging cylindrical shock waves in air. Exps. Fluids 5, 325-322.

Whitham, G. B. 1974 Linear and Nonlinear Waves. Wiley.

Zabusky, N., Zeng, S., Ray, J. \& Samtaney, R. 1997 Vortex projectiles from shock-bubble interactions. In 6th Intl Workshop on Compressible Turbulent Mixing (ed. G. Jourdan \& L. Houas), pp. 539-544. Imprimerie Caractère, Marseille. 